\title{
Investigation of transverse motion of concealed scatterer using correlation of speckled speckles from static surface layer
}

Nair, Gokul G.; Vinu, R.V.; Naik, Dinesh N.; Jakobsen, Michael Linde; Hanson, Steen Grüner; Singh, Rakesh Kumar

\section{Published in:}

Proceedings of SPIE

Link to article, DOI:

$10.1117 / 12.2320481$

Publication date:

2018

Document Version

Publisher's PDF, also known as Version of record

Link back to DTU Orbit

Citation (APA):

Nair, G. G., Vinu, R. V., Naik, D. N., Jakobsen, M. L., Hanson, S. G., \& Singh, R. K. (2018). Investigation of transverse motion of concealed scatterer using correlation of speckled speckles from static surface layer. In Proceedings of SPIE (Vol. 10750). [107500A] SPIE - International Society for Optical Engineering. Proceedings of SPIE - The International Society for Optical Engineering https://doi.org/10.1117/12.2320481

\section{General rights}

Copyright and moral rights for the publications made accessible in the public portal are retained by the authors and/or other copyright owners and it is a condition of accessing publications that users recognise and abide by the legal requirements associated with these rights.

- Users may download and print one copy of any publication from the public portal for the purpose of private study or research.

- You may not further distribute the material or use it for any profit-making activity or commercial gain

- You may freely distribute the URL identifying the publication in the public portal 


\section{Investigation of transverse motion of concealed scatterer using correlation of speckled speckles from static surface layer}

Gokul G. Nair, Vinu R.V., Dinesh N. Naik, Michael Linde Jakobsen, Steen G. Hanson, et al.

Gokul G. Nair, Vinu R.V., Dinesh N. Naik, Michael Linde Jakobsen, Steen G. Hanson, Rakesh Kumar Singh, "Investigation of transverse motion of concealed scatterer using correlation of speckled speckles from static surface layer," Proc. SPIE 10750, Reflection, Scattering, and Diffraction from Surfaces VI, 107500A (4 September 2018); doi: 10.1117/12.2320481

Event: SPIE Optical Engineering + Applications, 2018, San Diego, California, United States 


\title{
Investigation of Transverse Motion of Concealed Scatterer Using Correlation of Speckled Speckles From Static Surface Layer
}

\author{
Gokul G. Nair ${ }^{\text {a }}$, Vinu R.V ${ }^{\mathrm{b}}$, Dinesh N. Naik ${ }^{\mathrm{a}}$, Michael Linde Jakobsen ${ }^{\mathrm{c}}$, Steen G. Hanson ${ }^{\mathrm{c}}$, \\ and Rakesh Kumar Singh ${ }^{\mathrm{a}, \mathrm{d}}$

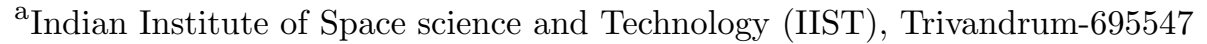 \\ ${ }^{\mathrm{b}}$ Center for Optical Research and Education (CORE), Utsunomiya University, Tochigi, 321-8585 JAPAN \\ ${ }^{c}$ DTU Fotonik, Department of Photonics Engineering, DK-4000 Roskilde, Denmark \\ ${ }^{\mathrm{d}}$ Department of Physics, Indian Institute of Technology (BHU), Varanasi, Uttar Pradesh, India
}

\begin{abstract}
Speckle-based techniques have noteworthy applications in the field of material science, surface characterization, determining mechanical displacements, biological activity in diffuse layer, imaging through turbid layer etc. The passage of coherent light through a diffuse layer generates a random speckle pattern, which have the inherent feature of carrying information associated with the diffuse layer. Dynamic laser speckle associated with the displacements of scattering surface has prominent impacts in the study of biological activity inside the diffuse layer. Investigations are progressing in the direction of exploring the dynamical properties associated with speckled speckles and its applications in imaging and characterization scenarios. In this work, we theoretically and experimentally study the dynamical properties of speckles through a static scattering layer using intensity correlation. The displacement (transverse or angular) produced in the concealed scatterer generates the dynamic speckle pattern which is observed through a second static diffuser. We expect to find applications of this investigation into the tracking objects hidden in the diffuse layer, measuring biological activity in diffuse layer, displacement measurements, etc.
\end{abstract}

Keywords: Speckle Correlation, Speckled Speckles, Velocity measurement, Bio Speckles, Imaging through turbid medium, Statistical Optics

\section{INTRODUCTION}

Speckle is a granular pattern that appears when highly coherent light is scattered by a random structure. ${ }^{1}$ In contrast to efforts to suppress speckle to improve imaging quality, there have also been approaches to exploit speckle patterns as a means of gathering physical information about a target. This field of research, known as speckle metrology, enables the measurement of various physical quantities such as vibration, roughness, and velocity. At the same time, speckle has also been used to measure the optical characteristics of light, including wavelengths and images. ${ }^{2}$ The use of granular structure named speckles that arise when coherent light is scattered from a rough surface and the intensity is probed has been extensively studied ${ }^{1}$ When the scattering surface is undergoing a displacement, the observed speckle pattern, itself, will show dynamic properties. This fact has been used intensively for various applications. ${ }^{3}$ Of special interest for the present evaluation is the determination of velocity of structures buried in a diffuse medium, here modeled as an illuminated dynamic structure giving rise to speckle but buried behind a diffuse screen. The issue of speckled speckles has previously been addressed. Françon et al. ${ }^{4}$ have experimentally shown how minute displacements can be monitored, while Iwai and Asakura ${ }^{5}$ experimentally have investigated triply-scattered speckles for displacement measurement. Here, the incident light first passed a diffuser, after which the speckled light hit the dynamic structure, and the scattered light passed the first diffuser once more and was detected. Also in the case of laser eye safety, the issue of speckled speckles is of importance ${ }^{6}$. In early investigations of the phenomenon of speckled speckles, experiments

Further author information: (Send correspondence to Gokul G. Nair)

Gokul G. Nair: E-mail: gokulgnair77755@gmail.com, Telephone: +91-9495400121

Reflection, Scattering, and Diffraction from Surfaces VI, edited by Leonard M. Hanssen, Proc. of SPIE Vol. 10750, 107500A · @ 2018 SPIE · CCC code: 0277-786X/18/\$18 · doi: 10.1117/12.2320481 
were conducted, in which the object was displaced with a constant velocity, and the spectral temporal content of the doubly scattered light was examined, especially with respect to its correlation decay time, ${ }^{5} \cdot{ }^{7-12}$ In this case, the distinction between speckle decorrelation ("boiling") and speckle displacement were mixed with the speckle size, giving little information on each individual component.

A specific reason for many of these investigations has been the investigation of optically based systems for probing subcutaneous blood flow. Ruth ${ }^{13}$ has very early shown how it is possible based on the dynamical speckled speckle pattern to provide information on the embedded blood flow. Of special interest in this evaluation was the exclusion of the speckle dynamics from the inadvertent movement of the skin, in order to enhance and separate the information from the blood flow. Later, investigations have been conducted showing measurements of blood flow ${ }^{14,15}$ and for measuring skin thickness, as well. ${ }^{16}$

In the following, we will sketch the derivation of speckle size, decorrelation length, and gearing of speckle displacement with relation to object displacement in case of speckled speckles. Furthermore, we will present analytical expressions in case the two diffusers are separated by free space, and the speckles arising from the second, and static, diffuser are observed after propagation in free space. The expressions will cover both the near field and the far field. No polarization effects will be included. The experimental findings which support the analytical results are also included.

\section{THEORETICAL DESCRIPTION}

We begin with the theoretical formulation of speckled speckles using field propagation through a pair of scattering media. Referring to the geometry of Figure[1], suppose that a scattering medium (diffuser) is placed a distance $z_{1}$ in front of a positive lens and is illuminated by monochromatic light. We represent the complex field immediately behind the object by $U_{0}(\xi, \eta)$. At a distance $z_{2}$ behind the lens there appears a field distribution that we represent by $U_{s}(u, v)$. In view of linearity of the wave propagation phenomenon, ${ }^{1}$ we can in all cases express the field $U_{s}$ by the following superposition integral:

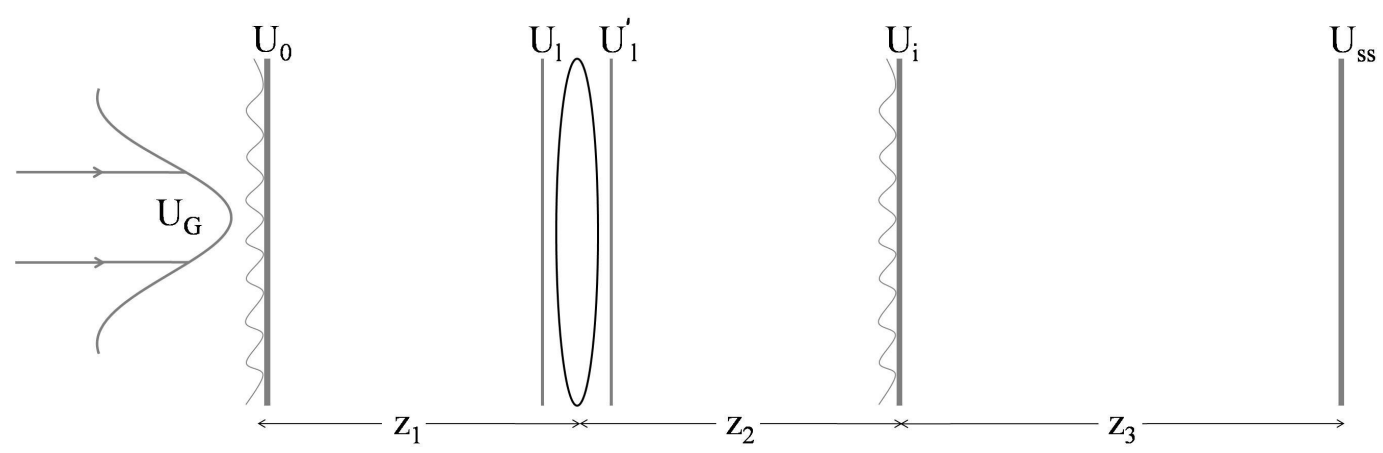

Figure 1. Geometry for formation of Speckled Speckles

$$
U_{s}(u, v)=\iint_{-\infty}^{\infty} G_{1}(u, v ; \xi, \eta) U_{0}(\xi, \eta) d \xi d \eta
$$

where $G_{1}(u, v ; \xi, \eta)$ is the Green's function connecting propagation between the first and second diffuser through an imaging lens. ${ }^{17}$ Thus the properties of the imaging system will be completely described if the Green's Function $G_{1}(u, v ; \xi, \eta)$ can be specified. If the optical system is to produce high-quality images, then $U_{s}$ must be as similar as possible to $U_{o}$. Equivalently, the impulse response should closely approximate a Dirac delta function,

$$
G_{1}(u, v ; \xi, \eta) \approx K \delta(u \pm M \xi, v \pm M \eta)
$$

where $\mathrm{K}$ is a complex constant, $\mathrm{M}$ represents the system magnification, and the plus and minus signs are included to allow for the absence or presence of image inversion, respectively. We shall therefore specify as the "image plane" that plane where (2) is most closely approximated. 
But, we know, $U_{0}(\xi, \eta)$ will be the complex field just after the first diffuser. Considering the Gaussian field as $U_{g}(\xi, \eta), U_{0}(\xi, \eta)$ is given by,

$$
U_{0}(\xi, \eta)=\iint_{-\infty}^{\infty} U_{g}\left(\xi^{\prime}, \eta^{\prime}\right) \exp \left[j \Phi\left(\xi^{\prime}, \eta^{\prime}\right)\right] \delta\left(\xi^{\prime}-\xi, \eta^{\prime}-\eta\right) d \xi^{\prime} d \eta^{\prime}
$$

Now, to find the Green's function $\left(G_{1}\right)$, let the object be a $\delta$ function (point source) at coordinates $(\xi, q)$. Then incident on the lens will appear a spherical wave diverging from the point $(\xi, q)$. The paraxial approximation to that wave is written,

$$
U_{l}(x, y)=\frac{1}{j \lambda z_{1}} \exp \left\{j \frac{k}{2 z_{1}}\left[(x-\xi)^{2}+(y-\eta)^{2}\right]\right\}
$$

After passage through the lens (focal length $\mathrm{f}$ ), the field distribution becomes

$$
U_{l}^{\prime}(x, y)=U_{l}(x, y) P(x, y) \exp \left[-j \frac{k}{2 f}\left(x^{2}+y^{2}\right)\right]
$$

Now, using the Fresnel diffraction equation to account for propagation over distance $z_{2}$, we have,

$$
G_{1}(u, v ; \xi, \eta)=\frac{1}{j \lambda z_{2}} \iint_{-\infty}^{\infty} U_{l}^{\prime}(x, y) \exp \left\{j \frac{k}{2 z_{2}}\left[(u-x)^{2}+(v-y)^{2}\right]\right\} d x d y
$$

where constant phase factors have been dropped. Combining (3), (4), and (5), and again neglecting a pure phase factor, yields the formidable result

$$
\begin{aligned}
& G_{1}(u, v ; \xi, \eta)=\frac{1}{\lambda^{2} z_{1} z_{2}} \exp \left[j \frac{k}{2 z_{2}}\left(u^{2}+v^{2}\right)\right] \exp \left[j \frac{k}{2 z_{1}}\left(\xi^{2}+\eta^{2}\right)\right] \\
& \times \iint_{-\infty}^{\infty} P(x, y) \exp \left[j \frac{k}{2}\left(\frac{1}{z_{1}}+\frac{1}{z_{2}}-\frac{1}{f}\right)\left(x^{2}+y^{2}\right)\right] \\
& \times \exp \left\{-j k\left[\left(\frac{\xi}{z_{1}}+\frac{u}{z_{2}}\right) x+\left(\frac{\eta}{z_{1}}+\frac{v}{z_{2}}\right)\right]\right\} d x d y
\end{aligned}
$$

Speckled Speckles generated at Observation plane $U_{s s}$ can be related to the Speckled field $U_{s}$ using another Green's function $G_{2}$. Where $G_{2}$ is defined as,

$$
G_{2}(\alpha, \beta ; u, v)=\frac{1}{j \lambda z_{3}} \exp \left\{j \frac{k}{2 z_{3}}\left[(\alpha-u)^{2}+(\beta-v)^{2}\right]\right\}
$$

Finally, using the Fresnel diffraction equation to account for propagation over distance $z_{3}$, we will get

$$
U_{s s}(\alpha, \beta)=\frac{1}{j \lambda z_{3}} \iint_{-\infty}^{\infty} U_{s}(u, v) \exp \left\{j \frac{k}{2 z_{3}}\left[(\alpha-u)^{2}+(\beta-v)^{2}\right]\right\} d u d v
$$

This gives the general information about the propagation. So, at the observation plane we get the intensity distribution $I_{s s}$ of complex field $U_{s} s$ as speckled speckles.

$$
I_{s s}(\alpha, \beta)=\left|U_{s s}(\alpha, \beta)\right|^{2}
$$

According to the experimental scheme in Figure [2], the following can be substituted.

$$
z_{1}=2 f
$$

Also, at exact imaging condition, where Equation [2] is valid,

$$
\begin{gathered}
z_{2}=2 f \\
z_{3}=l
\end{gathered}
$$




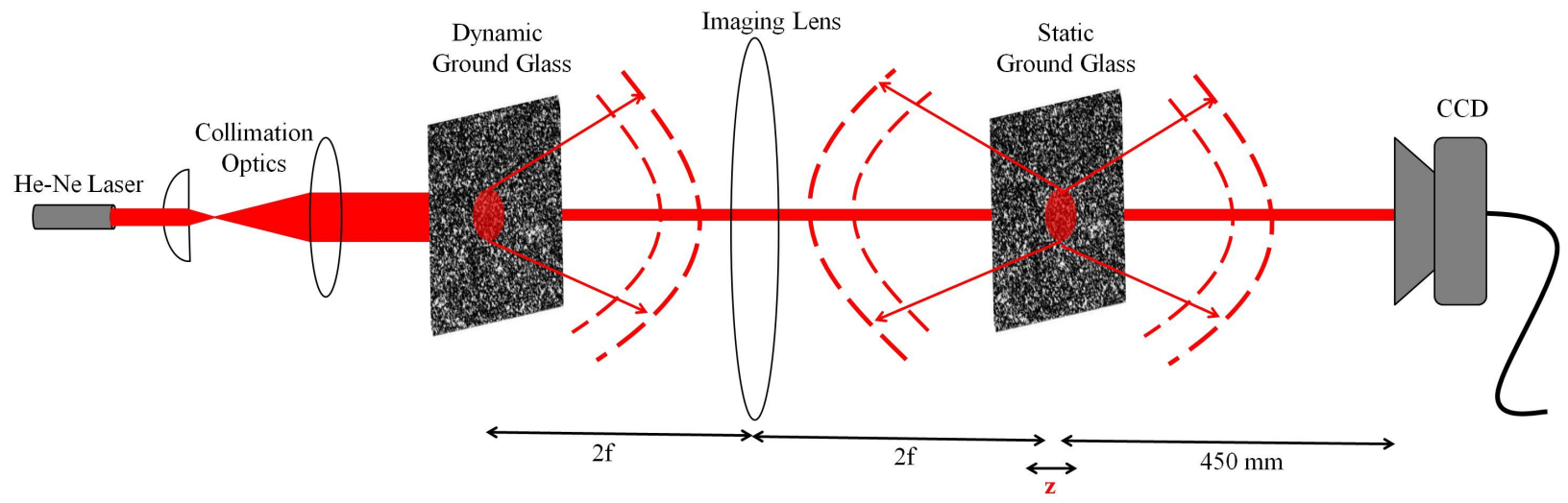

Figure 2. Experimental Scheme

In all other cases they will satisfy,

$$
z_{2}+z_{3}=\text { Constant }=L=2 f+l
$$

Now, for the experiment we will vary $z_{2}$ as $2 f \pm \Delta z$ and which will lead to $z_{3}=l \mp \Delta z$. This will always satisfy the Equation [13] for $z_{2}$ and $z_{3}$. Note that, $\Delta z$ will be zero at the imaging condition.

For each value of $\Delta z$, we will now move the first diffuser in lateral direction in steps of $\Delta \xi$.Remember the fact that the co-ordinate system will not shift here,but only the diffuser.

The new random phase introduced at each step by the shifting of diffuser is represented by,

$$
\Phi(\xi, \eta)=\Phi_{R}(\xi-\Delta \xi, \eta)
$$

Where, $\Phi_{R}(\xi, \eta)$ is phase distribution of the first scatterer at $\Delta \xi=0$. Then equation [3] changes as,

$$
U_{0}(\xi, \eta)=\iint_{-\infty}^{\infty} U_{g}\left(\xi^{\prime}, \eta^{\prime}\right) \exp \left[j \Phi_{R}\left(\xi^{\prime}-\Delta \xi^{\prime}, \eta^{\prime}\right)\right] \delta\left(\xi^{\prime}-\xi, \eta^{\prime}-\eta\right) d \xi^{\prime} d \eta
$$

Next, processing of the Speckled Speckles will takes place. We will execute intensity cross correlation of the resulting images. For each values of $\Delta z$ all the images with $\Delta \xi$ shift will be correlated with the reference image, which is taken when $\Delta \xi=0$.ie,

$$
C(\Delta \xi, \Delta z)=<\Delta I_{s s}(\alpha, \beta, \Delta \xi=0, \Delta z) \Delta I_{s s}(\alpha, \beta, \Delta \xi, \Delta z)>
$$

$C(\Delta \xi, \Delta z)$ is defined as the cross correlated output of the images in the observation plane.

$$
C_{R}(\Delta \xi, \Delta z)=<\Delta I_{s s}(\alpha, \beta, \Delta \xi=0, \Delta z) \Delta I_{s s}(\alpha, \beta, \Delta \xi=0, \Delta z)>
$$

$C_{R}(\Delta \xi, \Delta z)$ is the autocorrelated function of the Reference Intensity at $\Delta \xi=0$.Now, we will define $P$ as the position of peak intensity in the correlation output. Therefore, $\Delta P$ will be,

$$
\Delta P=P-P_{R}
$$

$P_{R}$ will be the position of the peak intensity in $C_{R}(\Delta \xi, \Delta z)$. Since each value of $\Delta \xi$ offers a corresponding $\Delta P$, we define the slope of graph plotted between $\Delta P$ and $\Delta \xi$ as Speckle Gearing, $G_{s}$.

$$
\text { Speckle Gearing, Gs }=\frac{\Delta P}{\Delta \xi}
$$




\section{EXPERIMENTAL ANALYSIS}

Using the scheme explained above, we conducted a series of experiments. The lens was having a focal length $\mathrm{f}$ of $100 \mathrm{~mm}$ and $\mathrm{l}$ was taken as $450 \mathrm{~mm} . z_{2}$ has been varied as $z_{2} \pm \Delta z$ where, $\Delta z$ ranges from $-5 \mathrm{~mm}$ to +20 $\mathrm{mm}$.(Here distances toward the lens from thesecond diffuser is taken as negative). For each value of $\Delta z$ the dynamic diffuser is varied from $-65 \mathrm{~nm}$ to $+65 \mathrm{~nm}$.

Each set of readings will be recorded for each value of $\Delta z$. Now, we will perform the intensity correlation of the Image field, for each image with respect to the reference image. The intensity correlation images are given in Figure 3.

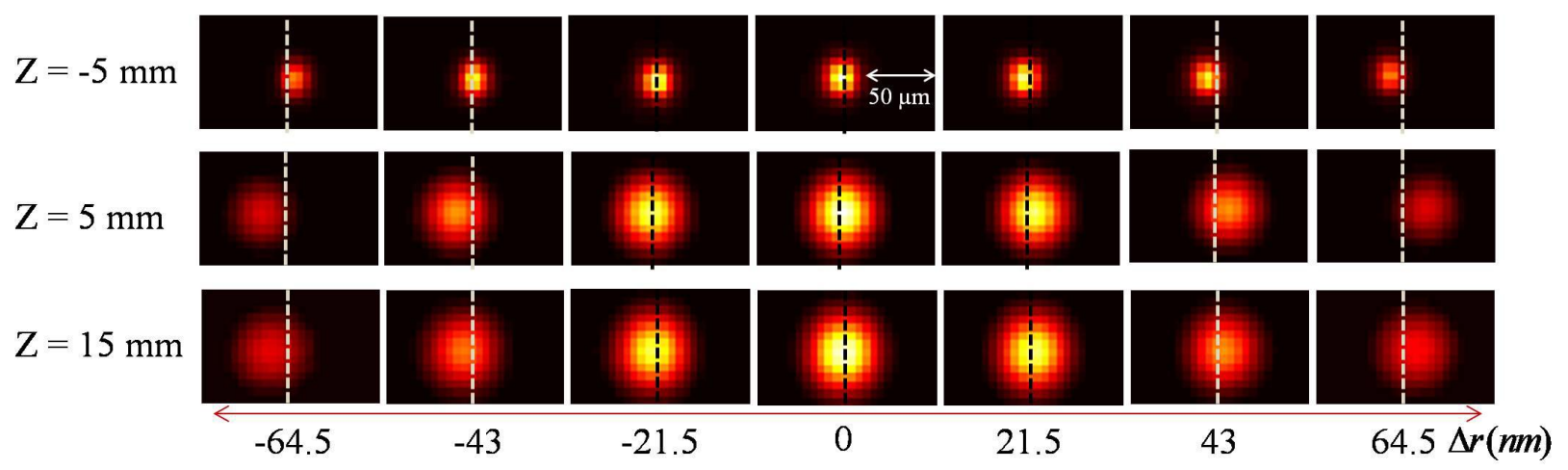

Figure 3. Cross Correlation of Image Intensities

The shift in the peak intensity position as well as decorrelation is visible from Figure [3]. The same trend continues for all values of $\Delta z$. Now we will plot graph between $\Delta P$ and $\Delta \xi$. The slope of the graph, ie. the speckle gearing is derived for each case.

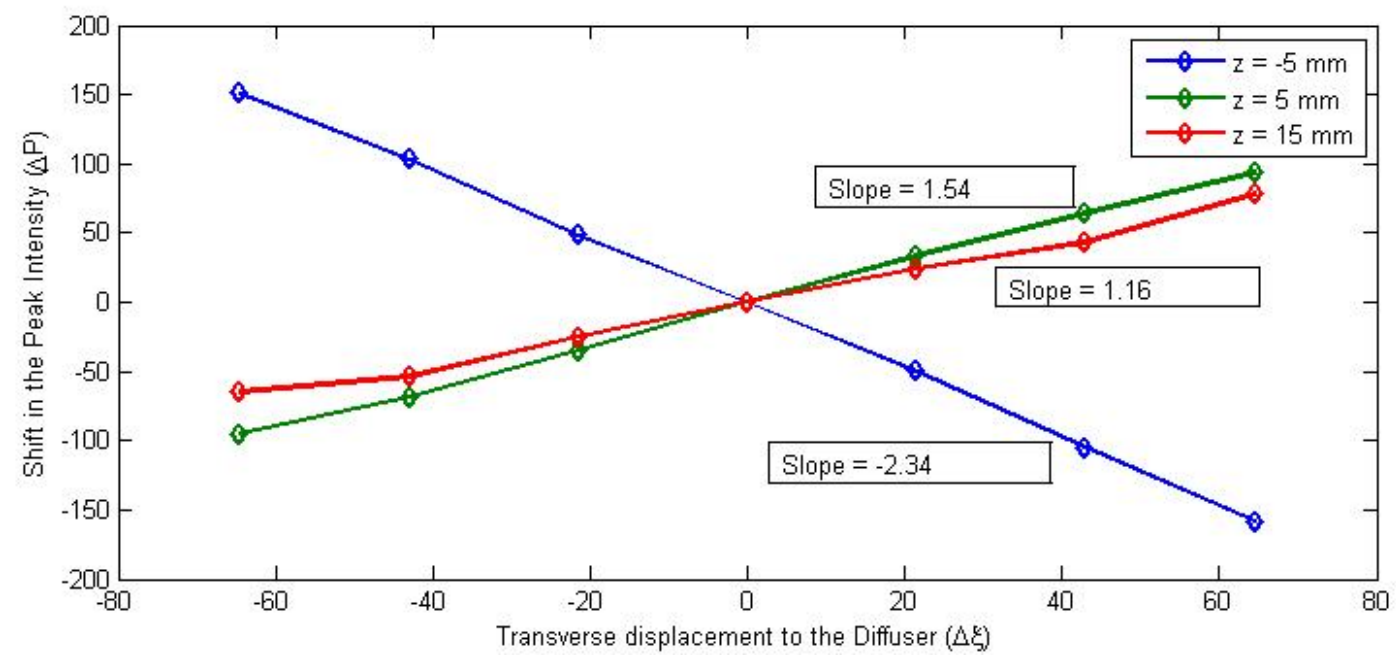

Figure 4. Graph plotted between $\Delta \xi$ and $\Delta P$ (Both are in $\mathrm{nm})$

As per the generalised ray- matrix formulation, when $B_{1}$ equals zero, the image of the first diffuser coincides with the second diffuser, and the gearing becomes zero. ${ }^{17}$ Accordingly, this corresponds to a z-value of $2.2 \mathrm{~mm}$. The decorrelation of the speckles revealed through decreasing intensity of peaks though remains similar, the correlation width varies as a function of $\mathrm{z}$. Most importantly, the speckle gearing provides the depth information as well as the flow of the concealed dynamic diffusing layer. 


\section{DISCUSSION}

The expressions for speckle dynamics have been derived and verified experimentally. The parameters such as Speckle size, Speckle Gearing are obtained in both methods. Since the far field propagation geometry is involved slight changes will be introduced to the equations when variations in the distance appear, which will lead to violation of Equation [2].

The results prove that using these properties depth information can be obtained, which will have application in flow measurements inside a diffuse layer. Furthermore, the investigation is important when we measure the blood flow in tissue, where scattering from the skin surface will act as the static diffuser and blood will act as the dynamic diffuser. However, in all the applications the Speckle size and Gearing will depend upon the distance between two surfaces and the motion of dynamic diffuser as well.

\section{ACKNOWLEDGMENTS}

Prof. Steen G. Hanson visited Indian Institute of Space Science and Technology (IIST) using the Visiting Lecturer Grant of IIST Student Chapter, which led to this collaborative work. We acknowledge SPIE for that opportunity and their continuous support all the time.

\section{REFERENCES}

[1] Goodman, J. W., [Speckle Phenomena in Optics], Roberts \& Company (2007).

[2] Kim, K., Yu, H., Lee, K., and Park, Y., "Universal sensitivity of speckle intensity correlations to wavefront change in light diffusers," Scientific Reports 7, 44435 (2017).

[3] H. J. Rabal, H. J. and Braga, R. A. J., "Dynamic laser speckle and applications," Anonymous (CRC Press) , pp. 272 (2008).

[4] M. Françon, P. K. and May, M., "Detection of small displacements with the help of an auxiliary diffuser," Opt. Commun. 12, 63-65 ((1974)).

[5] Iwai, T. and Shigeta, K., "Experimental study on the spatial correlation properties of speckled speckles using digital speckle photography," Jpn J Appl Phys Part 1 Regul Pap Short Note Rev Pap 29, 1099-1102 $((1990))$.

[6] Fried, D. L., "Laser eye safety: the implications of ordinary speckle statistics and of speckled-speckle statistics," J. Opt. Soc. Am. 71, 914-916 ((1981)).

[7] George, N. and Jain, A., "Speckle from a cascade of two diffusers," Opt. Commun. 15, 71-75 ((1975)).

[8] Iwai, T. and Asakura, T., "Dynamic properties of speckled speckles with relation to velocity measurements of a diffuse object," Opt. Laser Technol. 21, 31-36 ((1989)).

[9] Y. Liu, X. P. and Ma, S. N., "Some analyses for slow velocity measurement of the diffuser by using dynamic laser speckle," in International Conference on Optoelectronic Science and Engineering '90 Wang Da-Heng, ed.(Publ by Int Soc for Optical Engineering) (1990).

[10] Okamoto, T. and Asakura, T., "Detection of the object velocity using doubly-scattered dynamic speckles under gaussian-beam illumination," Journal of Modern Optics 38, 1821-1839 ((1991)).

[11] Okamoto, T. and Asakura, T., "Velocity dependence of image speckles produced by a moving diffuser under dynamic speckle illumination," Opt. Commun. 77, 113-120 ((1990)).

[12] Ruth, B., "Superposition of two dynamic speckle patterns," Journal of Modern Optics 39, 2421-2436 $((1992))$.

[13] Ruth, B., "Superposition of two dynamic speckle patterns. an application to non-contact blood flow measurements," Journal of Modern Optics 34, 257-273 ((1987)).

[14] Y. Liu, X. Zhang, X. S. S. M. M. Z. X. W. and Huang, Q., "Clinical studies of the biospeckle blood flow meter," in Lasers in Medicine and Dentistry: Diagnostics and Treatment, Khorana Brij M., Li Junheng, and Pankratov Michail M., eds. (1996).

[15] Ulyanov, S. S., "Using of speckled speckles in high-resolution measurement of bioflow," in Proceedings of the 1999 4th International Conference on Correlation Optics Anonymous (Society of Photo-Optical Instrumentation Engineers (1999). 
[16] W. Wuqi, F. Rulian, B. E. L. Y. Z. X. and Shining, M., "Application of correlation dimension of biospeckles to measuring skin thickness," in Proceedings of the 1999 Pacific Rim Conference on Lasers and Electro-Optcis (CLEO/PACIFIC Rim '99)Anonymous(IEEE) (1999).

[17] Hanson, S. G., Iversen, T. F. Q. F. Q., and Hansen, R. S., "Dynamic properties of speckled speckles," in [Speckle 2010: Optical Metrology], 7387, 738716, International Society for Optics and Photonics (2010). 